\title{
A new proof of fermat's last theorem
}

\begin{abstract}
For centuries, the proof of Fermat's last theorem using mathematical theory and techniques of his time has been a mystery for the entire mathematical community. Over the years, a large number of attempts have been made without so far a widely accepted proof that is based purely on mathematics that Fermat could have used himself. In the present work, we provide a novel approach to prove Fermat's famous Theorem. We assume that the theorem states a true proposition and we end up in a contradiction, thus concluding that the initial assumption cannot hold, emphasizing the value of a classical method of proof, such as the proof by contradiction. Our method is based on the concept of the limit, a well known topic even at Fermat's time, upon which the modern mathematical edifice was built.
\end{abstract}

Volume I Issue 3 - 2018

\author{
A Mazaris \\ University of Athens, Greece \\ Correspondence: A Mazaris, Gnosis Mathematical Research \\ Institute, 25, Agiou Antoniou str., 15235 Vrilissia, Greece, Tel \\ +3069 4002 5916, Email a.mazaris@yahoo.gr
}

Received: May 05, 2018 | Published: June 06, 2018

Keywords: fermat, fermat's last theorem, diophantine equations

\section{Introduction}

Fermat's Last Theorem (1637) is perhaps the single most famous mathematical problem of all times. Despite the extensive literature devoted to its proof, and its numerous applications in other field of science, it remained unsolved for nearly 4 centuries. Although finally proved in 1995 by A Wiles ${ }^{1}$ the Theorem never stopped being a challenge for the broader mathematical community, mainly because Wiles' proof was based on an extensive mathematical background that was not nearly available in Fermat's era. Over the years, a large number of attempts were unsuccessful, giving reasonable raise to concerns questioning whether Fermat had indeed come up with a general and concise proof, as he has claimed in his writings. Could Fermat have proven his Last Theorem? This is a question of both historical and mathematical value, regardless of the unambiguous significance of Wiles' modern proof.

In this study, we attempt to prove Fermat's Last Theorem using a novel approach that is based on the concept of limit, which was known in considerable depth back in the $16^{\text {th }}$ century, suggesting that it could have been close to Fermat's unrevealed proof. The work employs the well-know technique of the proof by contradiction, and is structured in 2 parts, leading to the final result.

\section{A novel approach using a classic method}

The Theorem states that: if $a, b, c$ is positive integers then there is no natural integer $n>2$. such that:

$$
x^{n}+y^{n}=z^{n}
$$

We will assume that Fermat's Theorem is valid, that is we accept that there are positive integers $a, b, c$ and a natural integer $n>2$ for which equation (1) is satisfied.

Let us consider the relationship:

$$
x^{n}+y^{n}=z^{n}
$$

where $x$ is a positive real number, $y$ a positive integer, and $z$ a positive real number. Also, let us assume that $x>a$ and $y>b$

We will prove that the above relationship is valid:

Let $y$ be a positive integer such that $y>b$ and $n$ a natural integer such that $n>2$.

Then the number $y^{n}$ is a positive integer number. Also, let $x$ be a positive real number such that $x>a$ and $n$ a natural integer such that $n>2$. Then the number $x^{n}$ is positive real number. If

$$
x^{n}+y^{n}=\lambda
$$

Then $\lambda$ is a positive real number as the sum of a positive integer number and a positive real number.

As a positive real number, $\lambda$ can be written in the form

$$
\lambda=(\sqrt[n]{\lambda})^{n}
$$

where $n$ is an integer number and $n>2$

Assigning $z=\sqrt[n]{\lambda}$ see Appendix A yields:

$$
x^{n}+y^{n}=z^{n}
$$

where $z$ is a positive real number

For $z^{n}$ it holds that $z^{n}>c^{n}$ because:

Since $y>b$ it follows that

$$
y^{n}>b^{n}
$$

Similarly, $x>a$ implies that

$$
x^{n}>a^{n}
$$

Adding relations (2) and (3) yields:

$$
\begin{aligned}
& x^{n}+y^{n}>a^{n}+b^{n} \\
& z^{n}>c^{n}
\end{aligned}
$$

In addition to equation (1) that we assume to be true, we have now proved that:

$$
x^{n}+y^{n}=z^{n} \text { where } n>2
$$

Subtracting (1) from (4) we obtain a new equation:

$$
x^{n}-a^{n}+y^{n}-b^{n}=z^{n}-c^{n} \text { where } n>2
$$

As produced by equations (1) and (4), equation (5) applies only if equation (1) holds, given that equation (4) was proved valid.

Notice that we must not accept all the triples of the form: $(x, y, z)=(k a, k b, k c)$ where $k$ is any positive integer and $k>1$. If we accept these triples, from equation (5) we will have:

$$
(k a)^{n}-a^{n}+(k b)^{n}-b^{n}=(k c)^{n}-c^{n}
$$


where $n$ any positive integer number

$$
\begin{aligned}
& k^{n} a^{n}-a^{n}+k^{n} b^{n}-b^{n}=k^{n} c^{n}-c^{n} \\
& \left(k^{n}-1\right) a^{n}+\left(k^{n}-1\right) b^{n}=\left(k^{n}-1\right) c^{n}
\end{aligned}
$$

If $k^{n}-1 \neq 0$ where $n$ any positive integer number, then $k \neq 1$.

Dividing by $k^{n}-1$ yields $a^{n}+b^{n}=c^{n}$. Therefore, the use of the equation: $x^{n}+y^{n}=z^{n}$ becomes unavailing.

Note that we must also exclude all the Pythagorean triples, because if $(a, b, c)$ is a Pythagorean triple then all triples $(k a, k b, k c)$ where $k$ any positive integer, are also Pythagorean. We suppose that equation (5) is true because it results from equation (1) (Fermat's Theorem) which we have assumed it is true. We will show that equation (5) always leads to a mathematical contradiction, thus proving that the equation initially assumed to be true (in our case Fermat's Theorem) is false.

In the following section, we will show that equation (5) cannot be true, because it leads to a contradiction. This automatically means that equation (1) is a false statement, because equation (4) is true and equation (5) has resulted from (1) and (4). We follow a 2-part proofing process, with the final outcome presented in the results section.

\section{Appendix A}

\section{Theorem}

Every real number $a>0$ has $\mathrm{n}^{\text {th }}$ root $(\mathrm{n}>1)$

\section{Proof}

We consider the function

$$
\begin{aligned}
& P(x): P(x)=x^{n}-a \\
& P(0)=-a<0
\end{aligned}
$$

By using Bernoulli's inequality

$$
P(a+1)=(a+1)^{n}-a \geq a+1-a=1>0
$$

According to Bolzano's theorem (and as $P(x)$ is a continuous function in $P\left(x_{0}\right)=0$

there is $x_{0} \in(0, a+1)$ that satisfies $P\left(x_{0}\right)=0$

Therefore $x_{0}^{n}-a=0$ or $x_{0}^{n}=a$

\section{Part I}

In this section we will start from equation (5) (i.e. we assume equation (1) is valid) and we will show that

$$
e^{\frac{1}{y^{n}-b^{n}}}=\lim _{x \rightarrow a}\left(\frac{z^{n}-c^{n}}{y^{n}-b^{n}}\right) \overline{x^{n}-a^{n}}
$$

Equation (5) states that

$$
x^{n}-a^{n}+y^{n}-b^{n}=z^{n}-c^{n}
$$

Since $y>b$ we obtain $y^{n}>b^{n}$ and $y^{n}-b^{n}>0$.

Dividing by $y^{n}-b^{n}$ yields: $\frac{x^{n}-a^{n}}{y^{n}-b^{n}}+1=\frac{z^{n}-c^{n}}{y^{n}-b^{n}}$ and as $x>a$, we can further proceed as:

$x^{n}>a^{n} \Rightarrow x^{n}-a^{n}>0 \Rightarrow \frac{1}{x^{n}-a^{n}}>0$

Therefore

$$
\begin{aligned}
& \left(\frac{x^{n}-a^{n}}{y^{n}-b^{n}}+1\right)^{\frac{1}{x^{n}-a^{n}}}=\left(\frac{z^{n}-c^{n}}{y^{n}-b^{n}}\right)^{\frac{1}{x^{n}-a^{n}}} \\
& \left(1+\frac{x^{n}-a^{n}}{y^{n}-b^{n}}\right)^{\frac{1}{x^{n}-a^{n}}}=\left(\frac{z^{n}-c^{n}}{y^{n}-b^{n}}\right) \overline{x^{n}-a^{n}}
\end{aligned}
$$

The last equation can be written as:

$$
\left(1+\frac{1}{y^{n}-b^{n}} \cdot \frac{1}{\frac{1}{x^{n}-a^{n}}}\right)^{\frac{1}{x^{n}-a^{n}}}=\left(\frac{z^{n}-c^{n}}{y^{n}-b^{n}}\right) \overline{x^{n}-a^{n}}
$$

Both sides of equation (6) can be viewed as functions of variable $x$ (note that: $z^{n}-c^{n}=x^{n}-a^{n}+y^{n}-b^{n}$ )

Since $a$ is accumulation point for both sides of equation (6), it follows that:

$$
\lim _{x \rightarrow a}\left(1+\frac{1}{y^{n}-b^{n}} \cdot \frac{1}{\frac{1}{x^{n}-a^{n}}}\right)^{\frac{1}{x^{n}-a^{n}}}=\lim _{x \rightarrow a}\left(\frac{z^{n}-c^{n}}{y^{n}-b^{n}}\right)^{\frac{1}{x^{n}-a^{n}}}
$$

In equation (7), the term $\frac{1}{y^{n}-b^{n}}$ is a positive rational number.

Also, it holds that $\lim _{x \rightarrow a} \frac{1}{x^{n}-a^{n}}=+\infty$ because always $x^{n}-a^{n}>0$.

However, the definition of the exponential function:

Implies that

$$
e^{\omega}=\lim _{v \rightarrow \infty}\left(1+\frac{\omega}{v}\right)^{v}
$$

$$
e^{\omega}=\lim _{x \rightarrow a}\left(1+\frac{\omega}{f(x)}\right)^{f(x)}
$$

Where $f(x)$ is a function of variable $x$ and $\lim f(x)=+\infty$

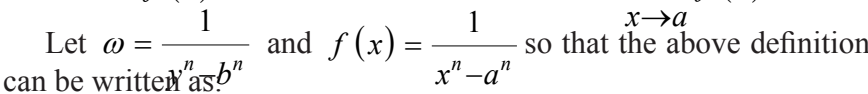

$$
e^{\omega}=\lim _{x \rightarrow a}\left(1+\frac{\omega}{f(x)}\right)^{f(x)} \text { with } D(f)=(a,+\infty)
$$

Substituting the above into equation (7) yields:

$$
e^{\frac{1}{y^{n}-b^{n}}}=\lim _{x \rightarrow a}\left(\frac{z^{n}-c^{n}}{y^{n}-b^{n}}\right) \overline{x^{n}-a^{n}}
$$




\section{Part 2}

In this section we will start again from equation (5) (i.e. we assume equation (1) is valid) and we will show that

$$
e^{1}=\lim _{x \rightarrow a}\left(\frac{z^{n}-c^{n}}{y^{n}-b^{n}}\right) \overline{\frac{1}{x^{n}-a^{n}}}
$$

Beginning from equation (5):

$$
x^{n}-a^{n}+y^{n}-b^{n}=z^{n}-c^{n} \quad(\text { with } n>2)
$$

Since $x^{n}-a^{n}>0$ :

$$
1+\frac{y^{n}-b^{n}}{x^{n}-a^{n}}=\frac{z^{n}-c^{n}}{x^{n}-a^{n}}
$$

In the last equation, the quantities $\frac{y^{n}-b^{n}}{x^{n}-a^{n}}$ and $\frac{z^{n}-c^{n}}{x^{n}-a^{n}}$ are positive real numbers so we can consider their natural logarithm $\ln \frac{y^{n}-b^{n}}{x^{n}-a^{n}}$ and $\ln \frac{z^{n}-c^{n}}{x^{n}-a^{n}}$

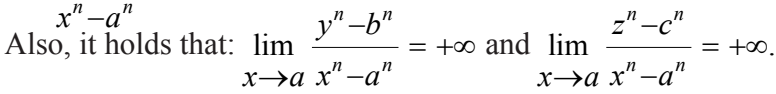

The quantities $\frac{y^{n}-b^{n}}{x^{n}-a^{n}}$ and $\frac{z^{n}-c^{n}}{x^{n}-a^{n}}$ are considered as functions of variable $x$, and $a$ is accumulation point for both of them.

Equation (9) now becomes:

$$
1+e^{\ln \frac{y^{n}-b^{n}}{x^{n}-a^{n}}}=e^{\ln \frac{z^{n}-c^{n}}{x^{n}-a^{n}}}
$$

Since $x^{n}-a^{n}>0$ implies that $\frac{1}{x^{n}-a^{n}}>0$, equation (10), and therefore equation (9), can be written as:

$$
1+e^{\left(x^{n}-a^{n}\right) \ln \left(\frac{y^{n}-b^{n}}{x^{n}-a^{n}}\right)^{\frac{1}{x^{n}-a^{n}}}}=e^{\left(x^{n}-a^{n}\right) \ln \left(\frac{z^{n}-c^{n}}{x^{n}-a^{n}}\right) \overline{x^{n}-a^{n}}}
$$

while as previously stated:

$$
\begin{aligned}
& \lim _{x \rightarrow a} \frac{y^{n}-b^{n}}{x^{n}-a^{n}}=+\infty \text { and } \lim _{x \rightarrow a} \frac{z^{n}-c^{n}}{x^{n}-a^{n}}=+\infty \\
& \text { At the limit of } \mathrm{x} \rightarrow a \text { equation (11) becomes: }
\end{aligned}
$$

$$
\lim _{\substack{x \rightarrow a \\ \text { and }}} e^{\left(x^{n}-a^{n}\right) \ln \left(\frac{y^{n}-b^{n}}{x^{n}-a^{n}}\right)^{x^{n}-a^{n}}}=\lim _{x \rightarrow a} \frac{y^{n}-b^{n}}{x^{n}-a^{n}}=+\infty
$$$$
\lim _{x \rightarrow a} e^{\left(x^{n}-a^{n}\right) \ln \left(\frac{z^{n}-c^{n}}{x^{n}-a^{n}}\right) \frac{1}{x^{n}-a^{n}}}=\lim _{x \rightarrow a} \frac{z^{n}-c^{n}}{x^{n}-a^{n}}=+\infty
$$

According to the well known property of exponential function:

$$
\left.\lim _{x \rightarrow a} e^{\left(x^{n}-a^{n}\right) \ln \left(\frac{y^{n}-b^{n}}{x^{n}-a^{n}}\right) \frac{1}{x^{n}-a n}}=e^{\lim _{x \rightarrow a}\left[\left(x^{n}-a^{n}\right) \ln \left(\frac{y^{n}-b^{n}}{x^{n}-a^{n}}\right) \frac{1}{x^{n}-a^{n}}\right.}\right]
$$

Using the properties of limits we further get:

$$
e^{\lim _{x \rightarrow a}\left[\left(x^{n}-a^{n}\right) \ln \left(\frac{y^{n}-b^{n}}{x^{n}-a^{n}}\right)^{\frac{1}{x^{n}-a^{n}}}\right]} \lim _{=e^{x \rightarrow a}}\left(x^{n}-a^{n}\right) . \lim _{x \rightarrow a} \ln \left(\frac{y^{n}-b^{n}}{x^{n}-a}\right)^{\frac{1}{x^{n}-a^{n}}}
$$

The $\lim _{x \rightarrow a} \ln \left(\frac{y^{n}-b^{n}}{x^{n}-a^{n}}\right)^{\frac{1}{x^{n}-a^{n}}}$ is defined because $a$ is accumulation point for the function $\ln \left(\frac{y^{n}-b^{n}}{x^{n}-a^{n}}\right)^{\frac{1}{x^{n}-a^{n}}}$ (considered as function of
variable $x$ ).

Similarly we have:

$$
e^{\lim _{x \rightarrow a}\left[\left(x^{n}-a^{n}\right) \ln \left(\frac{z^{n}-c^{n}}{x^{n}-a^{n}}\right) \frac{1}{x^{n}-a^{n}}\right]}=e^{\lim _{x \rightarrow a}\left(x^{n}-a^{n}\right) \cdot \lim _{x \rightarrow a} \ln \left(\frac{z^{n}-c^{n}}{x^{n}-a^{n}}\right) \frac{1}{x^{n}-a^{n}}}
$$

Note the $\lim _{x \rightarrow a} \ln \left(\frac{z^{n}-c^{n}}{x^{n}-a^{n}}\right)^{\frac{1}{x^{n}-a^{n}}}$ is defined because $a$ is an \[ x \rightarrow a\left(x^{n}-a^{n}\right) \]
accumulation point for the function $\ln \left(\frac{z^{n}-c^{n}}{x^{n}-a^{n}}\right)^{\frac{1}{x^{n}-a^{n}}}$ (considered
as a function of variable $x$ ). Finally, applying the property of exponentiation: $e^{x y}=\left(e^{x}\right)^{y}$ we have:

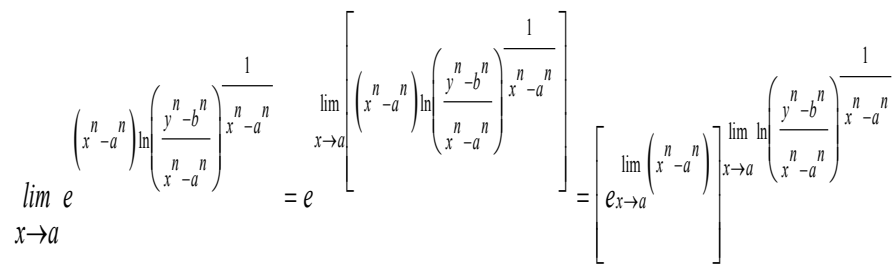

and

$\lim _{x \rightarrow a} e^{\left(x^{n}-a^{n}\right) \ln \left(\frac{z^{n}-c^{n}}{x^{n}-a^{n}}\right) \frac{1}{x^{n}-a^{n}}}=e^{\lim _{x \rightarrow a}\left[\left(x^{n}-a^{n}\right) \ln \left(\frac{z^{n}-c^{n}}{x^{n}-a^{n}}\right)^{\frac{1}{x^{n}-a^{n}}}\right]}=\left[\lim _{x \rightarrow a}\left(x^{n}-a^{n}\right)\right]^{\lim \ln \left(\frac{z^{n}-c^{n}}{x^{n}-a^{n}}\right)^{\frac{1}{x^{n}-a^{n}}}}$

Recall that through equations (12) and (13) we showed that the

limit of equations (16) and (17) is $+\infty$.
We will proceed by proving that $\ln \left(\frac{z^{n}-c^{n}}{x^{n}-a^{n}}\right) \frac{1}{x^{n}-a^{n}}$ 
It holds that:

$$
\lim _{x \rightarrow a} \frac{y^{n}-b^{n}}{x^{n}-a^{n}}=+\infty
$$

Also, it holds that $\lim _{x \rightarrow a}\left(x^{n}-a^{n}\right)=0$

therefore

$$
\lim _{x \rightarrow a} \frac{1}{x^{n}-a^{n}}=+\infty
$$

because $x^{n}-a^{n}$ is always positive.

Equation (18) implies that:

$\lim _{x \rightarrow a} \ln \frac{y^{n}-b^{n}}{x^{n}-a^{n}}=+\infty$ (20) see Appendix B

By multiplying equations (19) and (20) we obtain:

$$
\begin{aligned}
& \left(\lim _{x \rightarrow a} \frac{1}{x^{n}-a^{n}}\right) \cdot\left(\lim _{x \rightarrow a} \ln \frac{y^{n}-b^{n}}{x^{n}-a^{n}}\right)=(+\infty) .(+\infty) \\
& \lim _{x \rightarrow a}\left(\frac{1}{x^{n}-a^{n}} \cdot \ln \frac{y^{n}-b^{n}}{x^{n}-a^{n}}\right)=+\infty \\
& \lim _{x \rightarrow a} \ln \left(\frac{z^{n}-c^{n}}{x^{n}-a^{n}}\right)^{\frac{1}{x^{n}-a^{n}}}=+\infty
\end{aligned}
$$

Similarly, we get:

$$
\lim _{x \rightarrow a} \ln \left(\frac{z^{n}-c^{n}}{x^{n}-a^{n}}\right)^{\frac{1}{x^{n}-a^{n}}}=+\infty
$$

It holds that:

$$
\lim _{x \rightarrow a}\left(x^{n}-a^{n}\right)=e^{0}=1
$$

Thus,

equation (16) results $1^{+\infty}$ (in our case it holds that $1^{+\infty}=+\infty$ ) and equation (17) then results $1^{+\infty}$ (in our case it holds that $1^{+\infty}=+\infty$ ).

Generally $1^{+\infty}$ is an indeterminate form, but here we showed that $1^{+\infty}=+\infty$.

To facilitate the rest of the work, we write the outcome of equation (16) as:

$$
1^{x \rightarrow a} \ln \left(\frac{y^{n}-b^{n}}{x^{n}-a^{n}}\right) \overline{\frac{1}{x^{n}-a^{n}}}
$$

where

$$
\lim _{x \rightarrow a} \ln \left(\frac{y^{n}-b^{n}}{x^{n}-a^{n}}\right)^{\frac{1}{x^{n}-a^{n}}}=+\infty
$$

and the outcome of equation (17) as:

$$
1^{\lim _{x \rightarrow a} \ln \left(\frac{z^{n}-c^{n}}{x^{n}-a^{n}}\right) \overline{\frac{1}{x^{n}-a}}}
$$

Where

$$
\lim _{x \rightarrow a} \ln \left(\frac{z^{n}-c^{n}}{x^{n}-a^{n}}\right)^{\frac{1}{x^{n}-a^{n}}}=+\infty
$$

Therefore, equation (11) now gives:

$$
1+e^{\left(x^{n}-a^{n}\right) \ln \left(\frac{y^{n}-b^{n}}{x^{n}-a^{n}}\right) \overline{x^{n}-a n}}=e^{\left(x^{n}-a^{n}\right) \ln \left(\frac{y^{n}-b^{n}}{x^{n}-a^{n}}\right) \frac{1}{x^{n}-a^{n}}}
$$

and thus

$$
\lim _{x \rightarrow a}\left[1+e^{\left(x^{n}-a^{n}\right) \ln \left(\frac{y^{n}-b^{n}}{x^{n}-a^{n}}\right)^{\frac{1}{x^{n}-a^{n}}}}\right]=\lim _{x \rightarrow a} e^{\left(x^{n}-a^{n}\right) \ln \left(\frac{y^{n}-b^{n}}{x^{n}-a^{n}}\right) \frac{1}{x^{n}-a^{n}}}
$$

The both limits exist because $a$ is an accumulation point of the corresponding functions.

Finally:

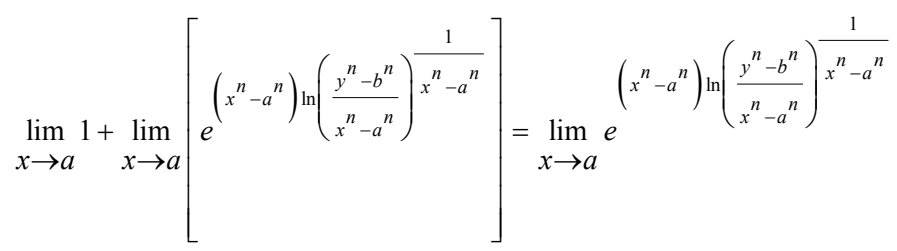

Using equations (16) and (17)

$1+\left[\lim _{x \rightarrow a}\left(x^{n}-a^{n}\right)\right]_{x \rightarrow a}^{\lim \ln \left(\frac{y^{n}-b^{n}}{x^{n}-a^{n}}\right)^{\frac{1}{x^{n}-a^{n}}}}=\left[\lim _{x \rightarrow a}\left(x^{n}-a^{n}\right)\right]_{x \rightarrow a}^{\lim \ln \left(\frac{z^{n}-c^{n}}{x^{n}-a^{n}}\right)^{\frac{1}{x^{n}-a^{n}}}}$

Using equations (21) and (22):

$1+1^{\lim _{x \rightarrow a} \ln \left(\frac{y^{n}-b^{n}}{x^{n}-a}\right)^{\frac{1}{x^{n}-a n}}}=1^{\lim \ln \left(\frac{z^{n}-c^{n}}{x^{n}-a^{n}}\right) \frac{1}{x^{n}-a^{n}}}$

where

$$
\begin{aligned}
& \lim _{x \rightarrow a} \ln \left(\frac{y^{n}-b^{n}}{x^{n}-a^{n}}\right)^{\frac{1}{x^{n}-a^{n}}}=+\infty \text { and } \\
& \lim _{x \rightarrow a} \ln \left(\frac{z^{n}-c^{n}}{x^{n}-a^{n}}\right)^{\frac{1}{x^{n}-a^{n}}}=+\infty
\end{aligned}
$$

Now, we will prove that any quantity of the form

$\lim f(x) \quad \lim f(x)$

$1^{x \rightarrow a} \quad$ where $\lim _{x \rightarrow a} f(x)=+\infty$ and $1^{x \rightarrow a}=+\infty$ can 
$\lim f(x)$

always get the value $e^{x \rightarrow a}$.

More specifically, we will show that:

$$
1^{\lim _{x \rightarrow a} f(x)}=e^{\lim _{x \rightarrow a} f(x)}
$$

where

$\lim _{x \rightarrow a} f(x)=+\infty$ and $1^{x \rightarrow a}=+\infty$

$x \rightarrow a$
We begin from the definition of the exponential function

$e=\lim _{x \rightarrow a}\left(1+\frac{1}{f(x)}\right)^{f(x)}$ and since $\lim _{x \rightarrow a} f(x)=+\infty$ we can write:

$$
\begin{gathered}
\frac{1}{\lim _{x \rightarrow a} f(x)}=1+\frac{1}{\lim _{x \rightarrow a} f(x)} \\
\frac{1}{\lim _{x \rightarrow a} f(x)}=\lim _{x \rightarrow a}\left[1+\frac{1}{f(x)}\right]
\end{gathered}
$$

If we raise both sides of the last equation to the power of $\left[\lim _{x \rightarrow a} f(x)\right]^{2}$, we have:

$$
\left[\frac{1}{\lim _{x \rightarrow a} f(x)}\right]^{\left[\lim _{x \rightarrow a} f(x)\right]^{2}}=\left\{\lim _{x \rightarrow a}\left[1+\frac{1}{f(x)}\right]\right\}^{\left[\lim _{x \rightarrow a} f(x)\right]^{2}}
$$

For the left-hand side of the last equation, the operation

$\frac{1}{\lim _{x \rightarrow a} f(x)} \cdot\left[\lim _{x \rightarrow a} f(x)\right]^{2}=\frac{\left[\lim _{x \rightarrow a} f(x)\right]^{2}}{\lim _{x \rightarrow a} f(x)}=\frac{\lim _{x \rightarrow a} f(x)^{2}}{\lim _{x \rightarrow a} f(x)}=\lim _{x \rightarrow a} \frac{f(x)^{2}}{f(x)}=\lim _{x \rightarrow a} f(x)$

is permitable $(f(x) \neq 0)$ according to the properties of limits.

Hence:

$$
\begin{aligned}
\lim _{x \rightarrow a} f(x) & =\lim _{x \rightarrow a}\left[1+\frac{1}{f(x)}\right]\left[\lim _{x \rightarrow a} f(x)\right]^{2} \\
\lim ^{x \rightarrow a} f(x) & =\left[\lim _{x \rightarrow a}\left[1+\frac{1}{f(x)}\right]^{\lim f(x)}\right]_{x \rightarrow a}^{\lim f(x)}
\end{aligned}
$$

$$
\begin{aligned}
& \lim _{x \rightarrow a}\left[1+\frac{1}{f(x)}\right]^{f(x)}=\lim _{x \rightarrow a} e^{\ln \left[1+\frac{1}{f(x)}\right]^{f(x)}}=e^{\lim _{x \rightarrow a} \ln \left[1+\frac{1}{f(x)}\right]^{f(x)}=e^{x \rightarrow a}\left[f(x) \ln \left[1+\frac{1}{f(x)}\right]\right]}= \\
& \left.=e^{\lim _{x \rightarrow a} f(x) \lim _{x \rightarrow a} \ln \left[1+\frac{1}{f(x)}\right]}=e^{\lim _{x \rightarrow a} f(x) \ln \left(\lim _{x \rightarrow a}\left[1+\frac{1}{f(x)}\right]\right)}=e^{\ln \left\{\lim _{x \rightarrow a}\left[1+\frac{1}{f(x)}\right]\right) \lim _{x \rightarrow a} f(x)}=\left\{\lim _{x \rightarrow a}\left[1+\frac{1}{f(x)}\right]\right\}\right\}^{\lim f(x)}
\end{aligned}
$$

Therefore:

$$
\lim _{x \rightarrow a}\left[1+\frac{1}{f(x)}\right]^{f(x)}=\left\{\lim _{x \rightarrow a}\left[1+\frac{1}{f(x)}\right]\right\}^{\lim f(x)}=e
$$

Substituting into (24) yields:

$$
1^{\lim _{x \rightarrow a} f(x)}=e^{\lim _{x \rightarrow a} f(x)}
$$

where

$\lim _{x \rightarrow a} f(x)=+\infty$ and $\lim _{1} f(x) a$
which we intended to show.

In equations (23) and (25) we consider as $f(x)$ the

quantities $\ln \left(\frac{y^{n}-b^{n}}{x^{n}-a^{n}}\right)^{\frac{1}{x^{n}-a^{n}}}$ and $\ln \left(\frac{z^{n}-c^{n}}{x^{n}-a^{n}}\right)^{\frac{1}{x^{n}-a^{n}}}$ which are functions of variable $x$ only (for $\ln \left(\frac{z^{n}-c^{n}}{x^{n}-a^{n}}\right)^{\frac{1}{x^{n}-a^{n}}}$ recall that:
$z^{n}-c^{n}=x^{n}-a^{n}+y^{n}-b^{n}$ ) and

$$
\lim _{x \rightarrow a} \ln \left(\frac{y^{n}-b^{n}}{x^{n}-a^{n}}\right)^{\frac{1}{x^{n}-a^{n}}}=+\infty, \quad \lim _{x \rightarrow a} \ln \left(\frac{z^{n}-c^{n}}{x^{n}-a^{n}}\right)^{\frac{1}{x^{n}-a^{n}}}=+\infty
$$

Also, it holds that

$$
1^{\lim \ln \left(\frac{y^{n}-b^{n}}{x^{n}-a}\right)^{\frac{1}{x^{n}-a^{n}}}}=+\infty, 1^{\lim \ln \left(\frac{z^{n}-c^{n}}{x^{n}-a}\right)^{\frac{1}{x^{n}-a^{n}}}}=+\infty
$$

Hence, equation (23) according to equation (25) gives:

$$
\begin{aligned}
& \lim _{1+1^{x \rightarrow a} \ln \left(\frac{y^{n}-b^{n}}{x^{n}-a^{n}}\right)^{\frac{1}{x^{n}-a n}}}=1^{\lim \ln \left(\frac{z^{n}-c^{n}}{x^{n}-a^{n}}\right)^{\frac{1}{x^{n}-a^{n}}}} \\
& \left.1+e^{\lim _{x \rightarrow a} \ln \left(\frac{y^{n}-b^{n}}{x^{n}-a}\right)^{\frac{1}{x^{n}-a n}}}=e^{\lim \ln \left(\frac{z^{n}-c^{n}}{x^{n}-a^{n}}\right)}\right)^{\frac{1}{x^{n}-a^{n}}}
\end{aligned}
$$

From the definition of the exponential function we have:

$$
e^{y}=\lim _{x \rightarrow a}\left[1+\frac{y}{f(x)}\right]^{f(x)} \text { where } \lim _{x \rightarrow a} f(x)=+\infty
$$


since $a$ is for $f(x)$ an accumulation point,

as a result:

$$
\lim _{\substack{x \rightarrow a \\ \text { or }}} e^{\frac{y}{f(x)}}=\lim _{x \rightarrow a}\left[1+\frac{y}{f(x)}\right] \text { where } \lim _{x \rightarrow a} f(x)=+\infty
$$$$
e^{\lim _{x \rightarrow a} \frac{y}{f(x)}}=1+\lim _{x \rightarrow a} \frac{y}{f(x)} \text { where } \lim _{x \rightarrow a} f(x)=+\infty
$$

The following equation:

$$
e^{\lim _{x \rightarrow a} \frac{y}{f(x)}}=1+\lim _{x \rightarrow a} \frac{y}{f(x)} \text { where } \lim _{x \rightarrow a} f(x)=+\infty
$$

is true in two cases:

$$
\begin{aligned}
& \text { a. When } \lim _{x \rightarrow a} \frac{y}{f(x)}=0 \\
& \text { b. When } \lim _{x \rightarrow a} \frac{y}{f(x)}=+\infty
\end{aligned}
$$

Hence $y$ can take any value, provided that any of the 2 conditions stated above is preserved.

If we assume $y=[f(x)]^{2}$ we have:

$$
\lim _{x \rightarrow a} \frac{y}{f(x)}=\lim _{x \rightarrow a} \frac{[f(x)]^{2}}{f(x)}=\lim _{x \rightarrow a} f(x)=+\infty
$$

So the value $y=[f(x)]^{2}$ is acceptable and the equation (27) now becomes:

$$
e^{\lim _{x \rightarrow a} f(x)}=1+\lim _{x \rightarrow a} f(x)
$$

Where $\lim _{x \rightarrow a} f(x)=+\infty$

This equation results directly from the definition of the exponential function and is applicable to any function $f(x)$ that satisfies $\lim f(x)=+\infty$

$\underset{\text { We will now apply equation (28) to equation (26) }}{x \rightarrow a}$

Note that we consider $\ln \left(\frac{y^{n}-b^{n}}{x^{n}-a^{n}}\right)^{\frac{1}{x^{n}-a^{n}}}$ and the $\ln \left(\frac{z^{n}-c^{n}}{x^{n}-a^{n}}\right)^{\frac{1}{x^{n}-a^{n}}}$ as functions of $x$ and let us also point out that $\lim _{x \rightarrow a} \ln \left(\frac{z^{n}-c^{n}}{x^{n}-a^{n}}\right)^{\frac{1}{x^{n}-a^{n}}}=+\infty$ and $\lim _{x \rightarrow a} \ln \left(\frac{z^{n}-c^{n}}{x^{n}-a^{n}}\right)^{\frac{1}{x^{n}-a^{n}}}=+\infty$

From equation (26) we obtain:

$$
1+e^{\lim _{x \rightarrow a} \ln \left(\frac{y^{n}-b^{n}}{x^{n}-a^{n}}\right) \frac{1}{x^{n}-a n}}=e^{\lim _{x \rightarrow a} \ln \left(\frac{z^{n}-c^{n}}{x^{n}-a^{n}}\right)} \frac{1}{x^{n}-a^{n}}
$$

and in accordance with equation (28)

$$
\begin{aligned}
& 1+1+\lim _{x \rightarrow a} \ln \left(\frac{y^{n}-b^{n}}{x^{n}-a^{n}}\right) \frac{1}{x^{n}-a^{n}}=1+\lim _{x \rightarrow a} \ln \left(\frac{z^{n}-c^{n}}{x^{n}-a^{n}}\right) \frac{1}{x^{n}-a^{n}} \\
& 1=\lim _{x \rightarrow a} \ln \left(\frac{z^{n}-c^{n}}{x^{n}-a^{n}}\right)^{\frac{1}{x^{n}-a^{n}}}-\lim _{x \rightarrow a} \ln \left(\frac{y^{n}-b^{n}}{x^{n}-a^{n}}\right) \frac{1}{x^{n}-a^{n}} \\
& 1=\lim _{x \rightarrow a}\left[\ln \left(\frac{z^{n}-c^{n}}{x^{n}-a^{n}}\right) \frac{1}{x^{n}-a^{n}}-\ln \left(\frac{y^{n}-b^{n}}{x^{n}-a^{n}}\right) \frac{1}{x^{n}-a^{n}}\right] \\
& 1=\lim _{x \rightarrow a}\left[\ln \left(\frac{z^{n}-c^{n}}{y^{n}-b^{n}}\right) \frac{1}{x^{n}-a^{n}}\right]
\end{aligned}
$$

by applying a property of logarithms,

$$
1=\ln \lim _{x \rightarrow a}\left(\frac{z^{n}-c^{n}}{y^{n}-b^{n}}\right)^{\frac{1}{x^{n}-a^{n}}}
$$

and finally

$$
e^{1}=\lim _{x \rightarrow a}\left(\frac{z^{n}-c^{n}}{y^{n}-b^{n}}\right) \frac{1}{x^{n}-a^{n}}
$$

\section{Appendix B}

\section{Theorem}

$$
\lim _{f(x) \rightarrow+\infty} \ln f(x)=+\infty:
$$

\section{Proof}

If $M>0$ since

$$
f(x)>0: \ln f(x)>M \Leftrightarrow \ln f(x)>\ln e^{M} \Leftrightarrow f(x)>e^{M}
$$

So if we choose $X=e^{M}$

for every $f(x)>X$ we will have $\ln f(x)>M$

leading to $\lim \ln f(x)=+\infty$.

\section{Results}

After a sequence of logical steps, we have now reached a contradiction. More specifically:

In Part 1, we have assumed that equation (5) is true and therefore equation (1) is satisfied. Then we showed that:

$$
e^{\frac{1}{y^{n}-b^{n}}}=\lim _{x \rightarrow a}\left(\frac{z^{n}-c^{n}}{y^{n}-b^{n}}\right)^{\frac{1}{x^{n}-a^{n}}}
$$

Similarly, in Part 2 we have assumed that equation (5) is true, and therefore equation (1) is satisfied, and we showed that: 


$$
e^{1}=\lim _{x \rightarrow a}\left(\frac{z^{n}-c^{n}}{y^{n}-b^{n}}\right)^{\frac{1}{x^{n}-a^{n}}}
$$

Equations (8) and (29) imply that:

$$
e^{\frac{1}{y^{n}-b^{n}}}=e^{1}
$$

because the limit of a function is unique.

Thus: $y^{n}-b^{n}=1$

Or $y^{n}=b^{n}+1$ for $n>2$

Since $y>b$ (by choise) there exist a positive integer $k$ such as: $y=b+k$, which leads to

$$
\begin{gathered}
(b+k)^{n}=b^{n}+1 \\
\stackrel{\text { or } \quad(b+k) \cdot(b+k) \cdot \ldots \cdot(b+k)}{\stackrel{\mathrm{n} \text {-terms }}{\longrightarrow}}=b^{n}+1
\end{gathered}
$$

The left-hand side of the above equation produces the term $b^{n}$ and $k^{n}$. The equality necessarily holds for $k=1$, so we have: $(b+1)^{n}=b^{n}+1$

This equation applies only for $n=1$ which is a contradiction because we started with the supposition that $n>2$.

We have thus proved the following:

We have accepted that if $a, b, c$ are positive integers then there is natural integer $n>2$ such that:

$$
a^{n}+b^{n}=c^{n}
$$

and we ended up in a proven relation that contradicts our initial supposition, forcing us to conclude that it is wrong, meaning that if $n>2$ are positive integers then there is no natural integer $n>2$ such that:

as the Theorem states.

$$
a^{n}+b^{n}=c^{n}
$$

\section{Discussion and conclusion}

Combining the fundamental concept of the limit with a classical proofing method, as proof by contradiction is, we have provided a novel proof of Fermat's Last Theorem. Assuming that the equation introduced by the Theorem holds, and following a series of logical steps presented in 2 parts, we were able to reach a contradiction and thus prove the Theorem's proposition. The main novelty of our approach resides in the use of pre-modern mathematical concepts and techniques that have been known since Fermat's initial conjecture. As also stated in the introduction, there is an historic significance linked to a classic proof of the Theorem that we consider equally important to the well known existing proof.

To further illustrate the broad applicability of the Theorem, we select and present below 3 of its classic, direct applications.

1. According to Fermat's Last Theorem, there are no positive integers $a, b, c$ and a natural number $\mathrm{n}$ such that $n>2$ to satisfy the equation

$$
a^{n}+b^{n}=c^{n} .
$$

This means that there is no angle $A$ such that $\sin \mathrm{A}^{\mathrm{n}}+\cos \mathrm{A}^{\mathrm{n}}=1$ with n natural number such that $\mathrm{n} \geq 3$ and $a^{n}+b^{n}=c^{n}$ congruent numbers.

Proof:

If $a^{n}+b^{n}=c^{n}$ with $a, b, c$ positive integers and $n>2$, dividing by $c^{n}$ we have:

$$
\left(\frac{\mathrm{a}}{\mathrm{c}}\right)^{\mathrm{n}}+\left(\frac{\mathrm{b}}{\mathrm{c}}\right)_{\mathrm{a}}^{\mathrm{n}}=1
$$

where $\frac{\mathrm{a}}{\mathrm{c}}$ congruent number with $\frac{\mathrm{a}}{\mathrm{c}}<1$ and also $\frac{\mathrm{b}}{\mathrm{c}}$ congruent number with $\frac{\mathrm{b}}{\mathrm{c}}<1$.

By assigning $\sin \mathrm{A}=\frac{\mathrm{a}}{\mathrm{c}}$ and $\cos \mathrm{A}=\frac{\mathrm{b}}{\mathrm{c}}$ we have:

$$
\sin \mathrm{A}^{\mathrm{n}}+\cos \mathrm{A}^{\mathrm{n}}=1
$$

Then, according to Fermat's Last Theorem, there is no angle $A$ satisfying the above equation for $\mathrm{n} \geq 3$.

Sinusoidal functions are commonly used in Physics (in waves, oscillations and alternating current) as well as in Astrophysics (particle motion in combined magnetic-electric fields).

2. According to Fermat's Last Theorem, there are no positive integers $a, b, c$ and a natural number $\mathrm{n}$ such that $n>2$ to satisfy the equation

$$
a^{n}+b^{n}=c^{n} \text {. }
$$

This means that there is no elliptic curve Frey witch the form is

$$
\mathrm{y}^{2}=\mathrm{x}\left(\mathrm{x}+\mathrm{a}^{\mathrm{n}}\right)\left(\mathrm{x}-\mathrm{b}^{\mathrm{n}}\right)
$$

Elliptic curves ${ }^{2}$ are widely applied in elliptic curve cryptography (ECC) and integer factorization.

3. According to Fermat's Last Theorem, there are no positive integers $a, b, c$ and a natural number $\mathrm{n}$ such that $n>2$ to satisfy the equation

$$
a^{n}+b^{n}=c^{n} \text {. }
$$

For $n=3$, it follows that it is impossible to have a cube with a dimention (edge length) that is a natural number split into two cubes, each with natural number edges, and preserve its initial volume. This constraint is taken into account in industrial design, 3D printing and related software and can be viewed as the inability to expand the Pythagorean Theorem that revolutionized planar geometry, in 3D applications.

\section{Acknowledgements}

None.

\section{Conflict of interest}

The author declares that there is no conflict of interest.

\section{References}

1. Wiles A. Modular Elliptic Curves and Fermat's Last Theorem. Annals of Mathematics. 1995;141(3):443-551.

2. Washington LC. Elliptic Curves, Number Theory and Cryptography. London: Chapmanand Hall; 2008. 536p. 